\title{
Erratum to: Structural, electronic and magnetic properties of Fe and Co monatomic nanochains encapsulated in BN nanotube bundle
}

\section{Eur. Phys. J. Appl. Phys. (2013) 62: 30402}

Sirvan Naderi ${ }^{1}$, Masoud Shahrokhi ${ }^{1, a}$, Hamid Reza Noruzi ${ }^{2}$, Ahmad Gurabi², and Rostam Moradian ${ }^{3,4,5}$

${ }^{1}$ Young Researchers Club, Kermanshah Branch, Islamic Azad University, Kermanshah, Iran

2 Department of Physics, Islamic Azad University, Kermanshah Branch, Kermanshah, Iran

${ }^{3}$ Physics Department, Faculty of Science, Razi University, Kermanshah, Iran

${ }_{5}^{4}$ Nano Science and Technology Research Center, Razi University, Kermanshah, Iran

${ }^{5}$ Computational Physical Science Research Laboratory, Department of Nano-Science, Institute for Studies in Theoretical Physics and Mathematics (IPM), P.O. Box 19395-5531, Tehran, Iran

Received: 16 June 2013 / Accepted: 8 July 2013

Published online: 7 August 2013 - (C) EDP Sciences 2013

The correct list of authors for the article "Structural, electronic and magnetic properties of Fe and Co monatomic nanochains encapsulated in BN nanotube bundle" published in Eur. Phys. J. Appl. Phys. (2013) 62: 30402 is:

Sirvan Naderi ${ }^{1}$, Masoud Shahrokhi ${ }^{1, a}$, Hamid Reza Noruzi Ahmad Gurabi ${ }^{2}$, and Rostam Moradian ${ }^{3,4,5}$

1 Young Researchers Club, Kermanshah Branch, Islamic Azad University, Kermanshah, Iran

2 Department of Physics, Islamic Azad University, Kermanshah Branch, Kermanshah, Iran

${ }^{3}$ Physics Department, Faculty of Science, Razi University, Kermanshah, Iran

4 Nano Science and Technology Research Center, Razi University, Kermanshah, Iran

${ }^{5}$ Computational Physical Science Research Laboratory, Department of Nano-Science, Institute for Studies in Theoretical Physics and Mathematics (IPM), P.O. Box 19395-5531, Tehran, Iran

\footnotetext{
${ }^{a}$ e-mail: shahrokhimasoud37@gmail.com
} 